\title{
Influence of family size and birth order on risk of cancer: a population-based study
}

Melanie Bevier ${ }^{1 *}$, Marianne Weires ${ }^{1}$, Hauke Thomsen ${ }^{1}$, Jan Sundquist ${ }^{2,3}$ and Kari Hemminki ${ }^{1,2}$

\begin{abstract}
Background: Family size and birth order are known to influence the risk of some cancers. However, it is still unknown whether these effects change from early to later adulthood. We used the data of the Swedish FamilyCancer Database to further analyze these effects.

Methods: We selected over 5.7 million offspring with identified parents but no parental cancer. We estimated the effect of birth order and family size by Poisson regression adjusted for age, sex, period, region and socioeconomic status. We divided the age at diagnosis in two groups, below and over 50 years, to identify the effect of family size and birth order for different age periods.
\end{abstract}

Results: Negative associations for increasing birth order were found for endometrial, testicular, skin, thyroid and connective tissue cancers and melanoma. In contrast, we observed positive association between birth order and lung, male and female genital cancers. Family size was associated with decreasing risk for endometrial and testicular cancers, melanoma and squamous cell carcinoma; risk was increased for leukemia and nervous system cancer. The effect of birth order decreased for lung and endometrial cancer from age at diagnosis below to over 50 years. Combined effects for birth order and family size were marginally significant for thyroid gland tumors. Especially, the relative risk for follicular thyroid gland tumors was significantly decreased for increasing birth order.

Conclusion: Our findings suggest that the effect of birth order decreases from early to late adulthood for lung and endometrial cancer.

\section{Background}

Family size and birth order has been shown to have an effect on the risk of cancer through socioeconomic and biological factors. For example, local observations showed that lower birth weight as a consequence of higher birth order has been associated with a lower risk for breast cancer [1-3] and a lower risk for melanoma [2]. Negative association has also been detected in testicular cancer for children of higher birth order [4-6]. Higher birth order often implies higher parental age at conception, although the latter has not been reported to be a risk factor in some studies $[7,8]$ whereas in other studies there was an association found for cancer sites as breast and prostate cancer as well as childhood cancers [9-13]. Genetic diseases or cancer during childhood may shorten the reproduction phase of parents, which

\footnotetext{
* Correspondence: m.bevier@dkfz-heidelberg.de

'Division of Molecular Genetic Epidemiology, German Cancer Research Center (DKFZ), Im Neuenheimer Feld 580, D-69120 Heidelberg, Germany Full list of author information is available at the end of the article
}

could cause higher risk for individuals in families with fewer children [14]. This might lead to an association of early-onset cancer or childhood cancers within last born children. Risks for many types of cancer and morbidities have been associated with the socioeconomic status of an individual $[15,16]$. A decreasing risk for melanoma has been reported for increasing family size and was explained by limited affordability of sunny holidays and solarium visits of larger families $[14,17,18]$. Significant association between socioeconomic factors, family size and lung cancer has also been reported in a previous study based on the Swedish Family-Cancer Database $[19,20]$. Socioeconomic factors have been shown to influence obesity which is an important risk factor for endometrial and other cancers such as liver cancer, non-Hodgkin lymphoma and multiple myeloma [21-24]. The susceptibility to be overweight might be influenced by childhood environment and to be carried on to adulthood. Closer contact among family members of larger families has been shown to increase risk of infection
C Biomed Central

(c) 2011 Bevier et al; licensee BioMed Central Ltd. This is an Open Access article distributed under the terms of the Creative Commons Attribution License (http://creativecommons.org/licenses/by/2.0), which permits unrestricted use, distribution, and reproduction in any medium, provided the original work is properly cited. 
with Helicobacter pylori and other Helicobacter pylori related cancers $[2,25,26]$.

The goal of the present study was to systematically analyze the effects of birth order and family size on different types of cancer. As we used the newest update of the Swedish Family Cancer-Database we had more than 75,000 additional registered cancer cases as previous studies with 178,365 cases [19]. This updated version included a total of 254,697 of cancer cases in offspring. Excluding the offspring with affected parents resulted in a study population of 134,896 individuals. The larger number of cancer cases leaded to more robust estimates of associations and allowed us to include also more rare cancers, such as esophageal, eye, small intestinal, laryngeal and salivary gland cancers. Additionally, we were able to divide the age at diagnosis in two categories to quantify the effect of family size and birth order. Differences in risk estimates for individuals diagnosed before and after 50 years of age are useful for identifying the effects of birth order and family size during life. Both influence the childhood environmental and lifestyle. Our aim was to see whether these factors still have an influence on cancer during early and later adulthood and whether they change over time. We focused on that aspect, as this has not been analyzed in previous studies.

\section{Methods}

The Swedish Family-Cancer Database includes data from the Second-Generation Register, the Swedish Cancer Registry, the National Census and the Death Notification Registry covering all cancers from 1961 to 2006 according to the seventh revision of the International Classification of Diseases (ICD-7) [27]. Cancer studies (Family-Cancer Database) in the MigMed Database was approved by the Lund regional ethical committee on 8 / $12 / 2008$ (No. 409/2008) and with complementary approvals dated $9 / 1 / 2009$ and $1 / 22 / 2010$. A total of approximately 11 million individuals born after 1932 and their biological parents have been registered. The newest version assembled in 2009 contains also information on more than 1.2 million first and multiple primary cancers and in situ tumors [25]. For most of the individuals affected with cancer clinical information on tumors is also available. Additionally, residential and socioeconomic data are available from national censuses, which were carried out in 1960, 1970, 1980 and 1990.

The study included all individuals from the database with identified parents. Individuals having parents affected with cancer were excluded, because parental cancer history should not be taken into account to avoid any confounding effect. The risk through parental cancer cases will be separated from the risk caused by birth order or family size. This lead to a study population of 5,657,455 individuals where 134,896 individuals were affected with cancer. The birth order of every individual was defined through the mother's parity (grouped $1,2,3-4,5-17)$. The family size (grouped 1, 2, 3-4, 5-17) is defined as the number of children per mother. There were 669,137 singletons, more than 2.3 million offspring in families with two children, more than 2.2 million in families with three or four children and 437,907offspring in families with at least five children included in the study. To analyze the effects of birth order and family size for early and later adulthood the individuals were categorized for age at diagnosis less and over age 50.

A four-digit diagnostic code according to ICD-7 was used to identify cancer sites. Some cancers were grouped according to the following codes: upper aerodigestive tract (140-141.9, 143.0-148.9), rectum excluding anus (154-154.0, 154.8), liver and gallbladder (155.0156.9), lung (162.0-163), uterus (173-174), non-Hodgkin lymphoma (200-200.2, 202-202.2) and leukemia (204.0209.9).

Patients were followed up for a specific time interval depending on the information available for each person in the database. Follow up started at year of immigration, birth year, or start year of cancer registry (1961), whatever came latest, until year of diagnosis, death, emigration or end of the study (2006), whatever occurred first. Cancer cases and person-years were determined for every stratification class of the covariates included in the regression model. For the calculation of the relative risks and the corresponding 95\% confidence intervals, age at stopfollow (5-year-bands), sex, calendar period (1961-1985, 1986-1990, 1991-1995, 1996-2000, 20012006), region (big cities, northern Sweden, southern Sweden, other), socioeconomic status (agricultural worker, white-collar worker, other worker, professional, private, other), family size, and birth order have been included as covariates. The Genmod procedure in SAS (SAS version 9.2; SAS Institute, Cary, NC, USA) was used to fit the Poisson regression model. The cancer status (affected or not affected) is specified as a response variable, whereas the included covariates are explanatory variables. Parameter estimates $(\beta)$ are obtained by maximum likelihood estimation (MLE). These parameters are estimated numerically through an iterative fitting process. Cancer incidence for one group compared to the reference group was calculated to obtain relative risk estimates. P values for trend analysis were calculated using a Jonckheere-Terpstra test which is a nonparametric test for ordered differences among classes.

\section{Results}

The study included around 5.7 million individuals, of which 134,896 were affected with cancer. Table 1 gives an overview on the distribution of individuals and cancer cases by family size and birth order. In Table 2 the 
Table 1 Number of all individuals (and cancer cases) included in the study with respect to birth order and family size

\begin{tabular}{lccccc}
\hline $\begin{array}{l}\text { All families } \\
\text { Birth order }\end{array}$ & $\mathbf{1}$ & $\mathbf{2}$ & Family size & $\mathbf{5}$ & All \\
\hline 1 & $669,137(25,209)$ & $1,161,073(26,279)$ & $681,658(20,129)$ & $75,738(4,226)$ & $2,587,606(75,843)$ \\
2 & & $1,169265(18,325)$ & $686,860(15,439)$ & $76,402(3,543)$ & $1,932,527(37,307)$ \\
$3-4$ & & $851,555(13,395)$ & $154,668(5,315)$ & $1,006,223(18,170)$ \\
$5-17$ & & & $131,099(3,036)$ & $131,099(3,036)$ \\
All & & & & $437,907(16,120)$ & $5,657,455(134,896)$ \\
\hline
\end{tabular}

age at stop follow of the individuals included in the study is presented.

Table 3 shows relative risk estimates for birth order of all cancer sites analyzed separately for age at diagnosis below 50 years or above 50 years and combining both. Significant results at 5\% confidence level are written bold. A significantly increased relative risk for lung cancer $(\mathrm{RR}=1.08,99 \% \mathrm{CI}: 1.00-1.15)$ was found independent of the age at diagnosis. Birth order was associated with a decreased risk for endometrial and testicular cancers, melanoma, squamous cell skin cancer, and cancers of the thyroid gland and connective tissue. The relative risk for cervical cancer $(R R=0.83)$ was significantly decreased for individuals diagnosed below age 50 years.

The association between family size and cancer with singletons as reference is presented in Table 4. Family size was associated with a decreased relative risk for endometrial ( $R R=0.76$, 99\% CI: 0.70-0.84) and testicular cancer, squamous cell skin cancer and melanoma, whereas the opposite was observed for cancer of the nervous system and leukemia $(R R=1.20)$. For lymphoid leukemia there was a relative risk of 1.30 (95\% CI: 1.041.61) found for increasing family size (data not shown).

Table 2 Age at stopfollow of individuals (and cancer cases) included in the study

\begin{tabular}{lll}
\hline Age at stopfollow & All individuals & Cancer cases (\%) \\
\hline $0-4$ & 551,205 & $3,405(0.62)$ \\
$5-9$ & 508,742 & $2,277(0.45)$ \\
$10-14$ & 566,083 & $2,017(0.36)$ \\
$15-19$ & 585,185 & $2,753(0.47)$ \\
$20-24$ & 493,490 & $3,728(0,76)$ \\
$25-29$ & 454,454 & $5,161(1.14)$ \\
$30-34$ & 446,358 & $6,537(1.46)$ \\
$35-39$ & 411,925 & $8,014(1.95)$ \\
$40-44$ & 376,844 & $12,213(2.71)$ \\
$45-49$ & 296,309 & $13,364(4.51)$ \\
$50-54$ & 273,077 & $17,052(6.24)$ \\
$55-59$ & 276,905 & $20,842(7.53)$ \\
$60-64$ & 264,047 & $20,190(7.65)$ \\
$65-69$ & 178,049 & $14,516(8.15)$ \\
$70-$ & 109,678 & $4,827(4.40)$ \\
All & $5,657,455$ & $134,896(2.33)$ \\
\hline
\end{tabular}

In the stratified analysis for the age at diagnosis, the relative risk was increased for stomach cancer $(R R=$ 1.17) and decreased for cervical cancer $(R R=0.82)$ for individuals above age 50 at diagnosis.

The relative risk for cancer of the thyroid gland was marginally significantly decreased for birth order ( $\mathrm{P}$ for trend $=<.0001$; data not shown). The relative risk for the second born $(R R=0.87)$ was slightly higher than for the third or fourth born $(R R=0.82)$ or higher birth orders $(\mathrm{RR}=0.75)$.

The relative risk for testicular cancer was inversely associated with family size (data not shown). It was decreasing with increasing family size. There was no significant combined effect with birth order even if the trend seemed to show an inverse association. Table 5 shows significantly decreased relative risks with increasing birth order for papillary $(\mathrm{RR}=0.81,95 \% \mathrm{CI}$ : 0.72 $0.92)$ and follicular thyroid gland tumors $(R R=0.70$, 95\% CI: 0.52-0.94).

In the separate analysis for the two groups of age at diagnosis (Figure 1), family size was positively associated with stomach cancer for age of diagnosis of at least 50 years. We found highly significant results independent of the birth order (one child families: $R R=0.67$; two child families: $R R=0.73$; three or four child families: $R R=0.75)$. Most of the stomach cancer cases occur with an age of diagnosis of at least 50 years and the separate analysis for the younger age group shows no significant associations.

Lung cancer was positively associated with birth order for lower age at diagnosis. The relative risk ranged from 1.02 for second born to 1.38 for third or fourth born offspring to 1.39 for the fifth born. In contrast the relative risk was not significant for different birth order when the age at diagnosis was at least 50 years even if a trend was still noticeable.

Figure 2 shows a detailed analysis on endometrial cancer. There was an inverse association of risk and birth order for age at diagnosis below age 50. The relative risk ranges from 0.73 for second born to 0.74 for third or fourth born until 0.56 for at least fifth born with first born children are the reference. For age at diagnosis above 50 years the same trend was observed. Family size was negatively associated with relative risk for 
Table 3 Relative risks for birth order and age at diagnosis

\begin{tabular}{|c|c|c|c|c|c|c|c|c|c|c|}
\hline \multirow[b]{3}{*}{ Cancer site } & \multirow[b]{3}{*}{ (ICD-7 code) } & \multicolumn{3}{|c|}{ Age at diagnosis $<50$ years } & \multicolumn{3}{|c|}{ Age at diagnosis $\geq 50$ years } & \multirow[t]{2}{*}{ All } & \multirow[b]{2}{*}{ Birth order } & \multirow[b]{2}{*}{ Ref } \\
\hline & & & Birth order & Ref & & Birth order & Ref & & & \\
\hline & & $\mathrm{N}$ & $\begin{array}{l}\text { RR } \\
(95 \% \mathrm{Cl})\end{array}$ & $\mathrm{N}$ & $\mathbf{N}$ & $\begin{array}{l}\text { RR } \\
(95 \% \mathrm{Cl})\end{array}$ & $\mathbf{N}$ & $\mathrm{N}$ & $\begin{array}{l}\text { RR } \\
(95 \% \mathrm{Cl})\end{array}$ & $\mathbf{N}$ \\
\hline Upper aero-digestive tract & $(140+)$ & 402 & $\begin{array}{l}0.95 \\
(0.82-1.09) \\
\end{array}$ & 396 & 577 & $\begin{array}{l}1.09 \\
(0.98-1.21) \\
\end{array}$ & 807 & 979 & $\begin{array}{l}1.04 \\
(0.95-1.14) \\
\end{array}$ & 1203 \\
\hline Salivary glands & $(142)$ & 119 & $\begin{array}{l}\mathbf{0 . 7 9} \\
(0.63-0.99) \\
\end{array}$ & 141 & 59 & $\begin{array}{l}0.93 \\
(0.65-1.33) \\
\end{array}$ & 96 & 178 & $\begin{array}{l}0.85 \\
(0.70-1.04)\end{array}$ & 237 \\
\hline Esophagus & $(150)$ & 55 & $\begin{array}{l}0.89 \\
(0.59-1.37) \\
\end{array}$ & 52 & 248 & $\begin{array}{l}0.89 \\
(0.75-1.05)\end{array}$ & 435 & 303 & $\begin{array}{l}0.89 \\
(0.76-1.05)\end{array}$ & 487 \\
\hline Stomach & $(151)$ & 261 & $\begin{array}{l}1.03 \\
(0.84-1.26)\end{array}$ & 232 & 505 & $\begin{array}{l}1.07 \\
(0.95-1.19)\end{array}$ & 757 & 766 & $\begin{array}{l}1.06 \\
(0.94-1.20)\end{array}$ & 989 \\
\hline Small intestine & $(152)$ & 84 & $\begin{array}{l}0.94 \\
(0.71-1.24)\end{array}$ & 85 & 147 & $\begin{array}{l}1.12 \\
(0.92-1.36)\end{array}$ & 206 & 231 & $\begin{array}{l}1.07 \\
(0.91-1.26)\end{array}$ & 291 \\
\hline Colon & (153) & 938 & $\begin{array}{l}0.94 \\
(0.85-1.04) \\
\end{array}$ & 928 & 1532 & $\begin{array}{l}0.97 \\
(0.91-1.04)\end{array}$ & 2723 & 2470 & $\begin{array}{l}0.97 \\
(0.91-1.02)\end{array}$ & 3651 \\
\hline Rectum & $(154 / 1541)$ & 376 & $\begin{array}{l}1.03 \\
(0.85-1.26) \\
\end{array}$ & 343 & 1084 & $\begin{array}{l}1.03 \\
(0.96-1.11) \\
\end{array}$ & 1740 & 1460 & $\begin{array}{l}1.03 \\
(0.95-1.12) \\
\end{array}$ & 2083 \\
\hline Anus & $(1541)$ & 66 & $\begin{array}{l}1.08 \\
(0.77-1.50) \\
\end{array}$ & 56 & 85 & $\begin{array}{l}0.95 \\
(0.74-1.24) \\
\end{array}$ & 133 & 151 & $\begin{array}{l}1.02 \\
(0.83-1.24) \\
\end{array}$ & 189 \\
\hline Liver and gallbladder & $(155,156)$ & 217 & $\begin{array}{l}0.95 \\
(0.80-1.14) \\
\end{array}$ & 202 & 467 & $\begin{array}{l}0.92 \\
(0.82-1.04)\end{array}$ & 807 & 684 & $\begin{array}{l}0.94 \\
(0.85-1.04) \\
\end{array}$ & 1009 \\
\hline Pancreas & $(157)$ & 134 & $\begin{array}{l}0.88 \\
(0.69-1.12)\end{array}$ & 128 & 573 & $\begin{array}{l}0.93 \\
(0.81-1.07)\end{array}$ & 972 & 707 & $\begin{array}{l}0.93 \\
(0.84-1.03)\end{array}$ & 1100 \\
\hline Nose & $(160)$ & 49 & $\begin{array}{l}0.99 \\
(0.64-1.52)\end{array}$ & 46 & 47 & $\begin{array}{l}1.19 \\
(0.85-1.67)\end{array}$ & 67 & 96 & $\begin{array}{l}1.05 \\
(0.76-1.45)\end{array}$ & 113 \\
\hline Larynx & $(161)$ & 58 & $\begin{array}{l}0.82 \\
(0.61-1.10) \\
\end{array}$ & 71 & 153 & $\begin{array}{l}1.05 \\
(0.83-1.32)\end{array}$ & 227 & 211 & $\begin{array}{l}1.00 \\
(0.83-1.19)\end{array}$ & 298 \\
\hline Lung & $(162,163)$ & 589 & $\begin{array}{l}\mathbf{1 . 1 7} \\
(1.04-1.31)\end{array}$ & 454 & 2201 & $\begin{array}{l}1.03 \\
(0.97-1.09)\end{array}$ & 3343 & 2790 & $\begin{array}{l}\mathbf{1 . 0 8} \\
(1.02-1.13)\end{array}$ & 3797 \\
\hline Breast & $(170)$ & 5053 & $\begin{array}{l}1.03 \\
(0.82-1.28)\end{array}$ & 4778 & 5937 & $\begin{array}{l}0.98 \\
(0.95-1.01)\end{array}$ & 8751 & 10990 & $\begin{array}{l}1.00 \\
(0.91-1.11)\end{array}$ & 13529 \\
\hline Cervix & $(171)$ & 1609 & $\begin{array}{l}0.95 \\
(0.85-1.06)\end{array}$ & 1597 & 245 & $\begin{array}{l}\mathbf{0 . 8 3} \\
(0.71-0.97)\end{array}$ & 401 & 1854 & $\begin{array}{l}0.93 \\
(0.85-1.02)\end{array}$ & 1998 \\
\hline Endometrium & $(172)$ & 213 & $\begin{array}{l}\mathbf{0 . 7 2} \\
(0.64-0.81)\end{array}$ & 1042 & 321 & $\begin{array}{l}\mathbf{0 . 8 7} \\
(0.83-0.92)\end{array}$ & 1924 & 1255 & $\begin{array}{l}\mathbf{0 . 8 5} \\
(0.81-0.90)\end{array}$ & 2245 \\
\hline Uterus & $(173,174)$ & 140 & $\begin{array}{l}0.91 \\
(0.79-1.04)\end{array}$ & 151 & 106 & $\begin{array}{l}0.99 \\
(0.82-1.19)\end{array}$ & 166 & 246 & $\begin{array}{l}0.95 \\
(0.85-1.06)\end{array}$ & 317 \\
\hline Ovary & $(175)$ & 902 & $\begin{array}{l}0.98 \\
(0.88-1.08)\end{array}$ & 868 & 806 & $\begin{array}{l}1.03 \\
(0.95-1.13)\end{array}$ & 1177 & 1708 & $\begin{array}{l}1.01 \\
(0.94-1.09)\end{array}$ & 2045 \\
\hline Other female genital & $(176)$ & 128 & $\begin{array}{l}1.34 \\
(1.09-1.64)\end{array}$ & 90 & 101 & $\begin{array}{l}1.03 \\
(0.85-1.26)\end{array}$ & 138 & 229 & $\begin{array}{l}\mathbf{1 . 2 0} \\
(1.04-1.38)\end{array}$ & 228 \\
\hline Prostate & $(177)$ & 92 & $\begin{array}{l}1.04 \\
(0.83-1.31)\end{array}$ & 76 & 4552 & $\begin{array}{l}1.03 \\
(1.00-1.06)\end{array}$ & 7984 & 4644 & $\begin{array}{l}1.03 \\
(1.00-1.06)\end{array}$ & 8060 \\
\hline Testis & $(178)$ & 1668 & $\begin{array}{l}\mathbf{0 . 9 0} \\
(0.84-0.97)\end{array}$ & 1582 & 65 & $\begin{array}{l}0.78 \\
(0.60-1.02)\end{array}$ & 112 & 1733 & $\begin{array}{l}\mathbf{0 . 8 9} \\
(0.83-0.95)\end{array}$ & 1694 \\
\hline Other male genital & (179) & 71 & $\begin{array}{l}1.15 \\
(0.94-1.42)\end{array}$ & 60 & 81 & $\begin{array}{l}1.22 \\
(1.00-1.48)\end{array}$ & 98 & 152 & $\begin{array}{l}\mathbf{1 . 2 0} \\
(1.05-1.38)\end{array}$ & 158 \\
\hline Kidney & $(180)$ & 663 & $\begin{array}{l}1.00 \\
(0.90-1.10)\end{array}$ & 608 & 716 & $\begin{array}{l}0.99 \\
(0.89-1.10)\end{array}$ & 1184 & 1379 & $\begin{array}{l}0.99 \\
(0.92-1.06)\end{array}$ & 1792 \\
\hline Urinary bladder & $(181)$ & 489 & $\begin{array}{l}1.02 \\
(0.90-1.16)\end{array}$ & 486 & 1082 & $\begin{array}{l}0.94 \\
(0.88-1.02)\end{array}$ & 1964 & 1571 & $\begin{array}{l}0.97 \\
(0.91-1.04)\end{array}$ & 2450 \\
\hline Melanoma & (190) & 2789 & $\begin{array}{l}\mathbf{0 . 8 8} \\
(0.83-0.95)\end{array}$ & 2952 & 1310 & $\begin{array}{l}\mathbf{0 . 8 7} \\
(0.81-0.93)\end{array}$ & 2232 & 4099 & $\begin{array}{l}\mathbf{0 . 8 8} \\
(0.84-0.92)\end{array}$ & 5184 \\
\hline Squamous cell skin & (191) & 412 & $\begin{array}{l}0.97 \\
(0.84-1.12)\end{array}$ & 405 & 638 & $\begin{array}{l}\mathbf{0 . 8 7} \\
(0.79-0.95)\end{array}$ & 1309 & 1050 & $\begin{array}{l}\mathbf{0 . 9 0} \\
(0.83-0.98)\end{array}$ & 1714 \\
\hline
\end{tabular}


Table 3 Relative risks for birth order and age at diagnosis (Continued)

\begin{tabular}{|c|c|c|c|c|c|c|c|c|c|c|}
\hline Eye & (192) & 297 & $\begin{array}{l}1.04 \\
(0.89-1.22) \\
\end{array}$ & 243 & 88 & $\begin{array}{l}0.90 \\
(0.71-1.15) \\
\end{array}$ & 146 & 385 & $\begin{array}{l}1.01 \\
(0.88-1.15) \\
\end{array}$ & 389 \\
\hline Nervous system & (193) & 3730 & $\begin{array}{l}1.00 \\
(0.95-1.06) \\
\end{array}$ & 3214 & 1126 & $\begin{array}{l}0.99 \\
(0.92-1.07) \\
\end{array}$ & 1630 & 4856 & $\begin{array}{l}1.01 \\
(0.96-1.06) \\
\end{array}$ & 4844 \\
\hline $\begin{array}{l}\text { Thyroid } \\
\text { gland }\end{array}$ & (194) & 821 & $\begin{array}{l}\mathbf{0 . 8 6} \\
(0.79-0.95)\end{array}$ & 894 & 156 & $\begin{array}{l}\mathbf{0 . 7 9} \\
(0.64-0.97)\end{array}$ & 289 & 977 & $\begin{array}{l}\mathbf{0 . 8 5} \\
(0.78-0.92)\end{array}$ & 1183 \\
\hline Endocrine glands & (195) & 1013 & $\begin{array}{l}0.99 \\
(0.91-1.09)\end{array}$ & 961 & 568 & $\begin{array}{l}\mathbf{0 . 8 7} \\
(0.78-0.98)\end{array}$ & 952 & 1581 & $\begin{array}{l}0.95 \\
(0.89-1.02)\end{array}$ & 1913 \\
\hline Bone & (196) & 500 & $\begin{array}{l}1.06 \\
(0.94-1.20)\end{array}$ & 412 & 37 & $\begin{array}{l}1.11 \\
(0.69-1.79)\end{array}$ & 47 & 537 & $\begin{array}{l}1.08 \\
(0.95-1.22)\end{array}$ & 459 \\
\hline Connective tissue & (197) & 572 & $\begin{array}{l}\mathbf{0 . 8 8} \\
(0.78-0.98) \\
\end{array}$ & 576 & 161 & $\begin{array}{l}1.00 \\
(0.82-1.21)\end{array}$ & 254 & 733 & $\begin{array}{l}\mathbf{0 . 9 0} \\
(0.82-0.99)\end{array}$ & 830 \\
\hline Non-Hodgkin lymphoma & $(200,202)$ & 1330 & $\begin{array}{l}1.06 \\
(0.97-1.15) \\
\end{array}$ & 1115 & 1029 & $\begin{array}{l}0.99 \\
(0.91-1.08) \\
\end{array}$ & 1611 & 2359 & $\begin{array}{l}1.03 \\
(0.97-1.10) \\
\end{array}$ & 2726 \\
\hline Hodgkin lymphoma & (201) & 1001 & $\begin{array}{l}0.92 \\
(0.84-1.01)\end{array}$ & 947 & 76 & $\begin{array}{l}1.00 \\
(0.75-1.33)\end{array}$ & 109 & 1077 & $\begin{array}{l}0.93 \\
(0.85-1.01)\end{array}$ & 1056 \\
\hline Myeloma & (203) & 119 & $\begin{array}{l}0.94 \\
(0.74-1.19)\end{array}$ & 117 & 332 & $\begin{array}{l}1.04 \\
(0.89-1.21)\end{array}$ & 535 & 451 & $\begin{array}{l}1.02 \\
(0.89-1.15)\end{array}$ & 652 \\
\hline Leukaemia & $(204-209)$ & 2208 & $\begin{array}{l}0.98 \\
(0.90-1.06)\end{array}$ & 1910 & 709 & $\begin{array}{l}1.02 \\
(0.93-1.12)\end{array}$ & 1117 & 2917 & $\begin{array}{l}1.00 \\
(0.94-1.07)\end{array}$ & 3027 \\
\hline Other and unspecified sites & (other) & 406 & $\begin{array}{l}0.91 \\
(0.79-1.04) \\
\end{array}$ & 398 & 838 & $\begin{array}{l}\mathbf{0 . 8 9} \\
(0.80-0.99)\end{array}$ & 1505 & 1244 & $\begin{array}{l}\mathbf{0 . 9 1} \\
(0.84-0.98)\end{array}$ & 1903 \\
\hline Any site & (any) & 29574 & $\begin{array}{l}\mathbf{0 . 9 7} \\
(0.94-0.99)\end{array}$ & 27895 & 29479 & $\begin{array}{l}0.96 \\
(0.93-1.00)\end{array}$ & 47948 & 59053 & $\begin{array}{l}0.97 \\
(0.95-1.00)\end{array}$ & 75843 \\
\hline
\end{tabular}

Reference group: first born child. Bold type, $95 \% \mathrm{Cl}$ does not include 1.00. Poisson regression adjusted for age, sex, period, region, socioeconomic status. Ref.: reference, N:number of cases

Table 4 Relative risks for family size and age at diagnosis

\begin{tabular}{|c|c|c|c|c|c|c|c|c|c|c|}
\hline \multirow[b]{3}{*}{ Cancer site } & \multirow[b]{3}{*}{ (ICD-7 code) } & \multicolumn{3}{|c|}{ Age at diagnosis $<50$ years } & \multicolumn{3}{|c|}{ Age at diagnosis $\geq 50$ years } & \multirow[t]{2}{*}{ All } & \multirow[b]{2}{*}{ Family size } & \multirow[b]{2}{*}{ Ref } \\
\hline & & & Family size & Ref & & Family size & Ref & & & \\
\hline & & $\mathbf{N}$ & $\begin{array}{l}\text { RR } \\
(95 \% \mathrm{Cl}) \\
\end{array}$ & $N$ & $\mathbf{N}$ & $\begin{array}{l}\text { RR } \\
(95 \% \mathrm{Cl}) \\
\end{array}$ & $N$ & $\mathrm{~N}$ & $\begin{array}{l}\text { RR } \\
(95 \% \mathrm{Cl}) \\
\end{array}$ & $\mathbf{N}$ \\
\hline Upper aero-digestive tract & $(140+)$ & 694 & $\begin{array}{l}1.00 \\
(0.81-1.23)\end{array}$ & 104 & 1068 & $\begin{array}{l}0.96 \\
(0.84-1.09)\end{array}$ & 316 & 1762 & $\begin{array}{l}0.97 \\
(0.87-1.09)\end{array}$ & 420 \\
\hline Salivary glands & $(142)$ & 222 & $\begin{array}{l}0.85 \\
(0.62-1.17)\end{array}$ & 38 & 117 & $\begin{array}{l}0.87 \\
(0.61-1.24)\end{array}$ & 38 & 339 & $\begin{array}{l}0.87 \\
(0.68-1.10)\end{array}$ & 76 \\
\hline Esophagus & (150) & 85 & $\begin{array}{l}\mathbf{0 . 5 2} \\
(0.33-0.82)\end{array}$ & 22 & 528 & $\begin{array}{l}0.99 \\
(0.82-1.18)\end{array}$ & 155 & 613 & $\begin{array}{l}0.92 \\
(0.77-1.10)\end{array}$ & 177 \\
\hline Stomach & (151) & 423 & $\begin{array}{l}0.91 \\
(0.68-1.21)\end{array}$ & 70 & 1009 & $\begin{array}{l}\mathbf{1 . 1 7} \\
(1.02-1.34)\end{array}$ & 253 & 1432 & $\begin{array}{l}1.11 \\
(0.96-1.29)\end{array}$ & 323 \\
\hline Small intestine & (152) & 140 & $\begin{array}{l}0.76 \\
(0.53-1.09)\end{array}$ & 29 & 282 & $\begin{array}{l}1.14 \\
(0.91-1.44)\end{array}$ & 71 & 422 & $\begin{array}{l}1.03 \\
(0.84-1.27) \\
\end{array}$ & 100 \\
\hline Colon & (153) & 1633 & $\begin{array}{l}1.01 \\
(0.88-1.17) \\
\end{array}$ & 233 & 3213 & $\begin{array}{l}0.95 \\
(0.89-1.02) \\
\end{array}$ & 1042 & 4846 & $\begin{array}{l}0.97 \\
(0.91-1.03) \\
\end{array}$ & 1275 \\
\hline Rectum & $(154 / 1541)$ & 632 & $\begin{array}{l}1.13 \\
(0.78-1.65)\end{array}$ & 87 & 2152 & $\begin{array}{l}0.96 \\
(0.88-1.04)\end{array}$ & 672 & 2784 & $\begin{array}{l}0.98 \\
(0.88-1.09)\end{array}$ & 759 \\
\hline Anus & (1541) & 105 & $\begin{array}{l}0.93 \\
(0.59-1.48)\end{array}$ & 17 & 171 & $\begin{array}{l}1.03 \\
(0.75-1.41)\end{array}$ & 47 & 276 & $\begin{array}{l}1.01 \\
(0.79-1.30)\end{array}$ & 64 \\
\hline Liver and gallbladder & $(155,156)$ & 376 & $\begin{array}{l}1.23 \\
(0.92-1.65)\end{array}$ & 43 & 972 & $\begin{array}{l}0.95 \\
(0.83-1.08)\end{array}$ & 302 & 1348 & $\begin{array}{l}0.99 \\
(0.88-1.11)\end{array}$ & 345 \\
\hline Pancreas & (157) & 231 & $\begin{array}{l}1.00 \\
(0.72-1.40)\end{array}$ & 31 & 1176 & $\begin{array}{l}0.93 \\
(0.81-1.06)\end{array}$ & 369 & 1407 & $\begin{array}{l}0.94 \\
(0.84-1.05)\end{array}$ & 400 \\
\hline Nose & (160) & 85 & $\begin{array}{l}1.24 \\
(0.58-2.67)\end{array}$ & 10 & 95 & $\begin{array}{l}1.53 \\
(0.96-2.44)\end{array}$ & 19 & 180 & $\begin{array}{l}1.39 \\
(0.89-2.17)\end{array}$ & 29 \\
\hline Larynx & $(161)$ & 111 & $\begin{array}{l}1.09 \\
(0.64-1.86)\end{array}$ & 18 & 304 & $\begin{array}{l}1.15 \\
(0.91-1.45)\end{array}$ & 76 & 415 & $\begin{array}{l}1.15 \\
(0.90-1.47)\end{array}$ & 94 \\
\hline
\end{tabular}


Table 4 Relative risks for family size and age at diagnosis (Continued)

\begin{tabular}{|c|c|c|c|c|c|c|c|c|c|c|}
\hline Lung & $(162,163)$ & 926 & $\begin{array}{l}1.20 \\
(0.97-1.48) \\
\end{array}$ & 117 & 4254 & $\begin{array}{l}0.95 \\
(0.89-1.02) \\
\end{array}$ & 1290 & 5180 & $\begin{array}{l}0.99 \\
(0.93-1.05)\end{array}$ & 1407 \\
\hline Breast & $(170)$ & 8325 & $\begin{array}{l}0.91 \\
(0.70-1.20)\end{array}$ & 1506 & 11473 & $\begin{array}{l}0.98 \\
(0.95-1.02)\end{array}$ & 3215 & 19798 & $\begin{array}{l}0.96 \\
(0.86-1.08)\end{array}$ & 4721 \\
\hline Cervix & $(171)$ & 2782 & $\begin{array}{l}0.97 \\
(0.82-1.14)\end{array}$ & 424 & 488 & $\begin{array}{l}\mathbf{0 . 8 2} \\
(0.69-0.98)\end{array}$ & 158 & 3270 & $\begin{array}{l}0.93 \\
(0.82-1.05)\end{array}$ & 582 \\
\hline Endometrium & $(172)$ & 412 & $\begin{array}{l}\mathbf{0 . 6 7} \\
(0.55-0.81)\end{array}$ & 122 & 2145 & $\begin{array}{l}\mathbf{0 . 7 8} \\
(0.72-0.84)\end{array}$ & 821 & 2557 & $\begin{array}{l}\mathbf{0 . 7 6} \\
(0.71-0.82)\end{array}$ & 943 \\
\hline Uterus & $(173,174)$ & 258 & $\begin{array}{l}\mathbf{1 . 2 9} \\
(1.05-1.60)\end{array}$ & 33 & 208 & $\begin{array}{l}0.94 \\
(0.77-1.15)\end{array}$ & 64 & 466 & $\begin{array}{l}1.07 \\
(0.93-1.24) \\
\end{array}$ & 97 \\
\hline Ovary & $(175)$ & 1532 & $\begin{array}{l}0.98 \\
(0.84-1.16) \\
\end{array}$ & 238 & 1542 & $\begin{array}{l}0.99 \\
(0.89-1.11) \\
\end{array}$ & 441 & 3074 & $\begin{array}{l}0.99 \\
(0.90-1.09)\end{array}$ & 679 \\
\hline Other female genital & $(176)$ & 195 & $\begin{array}{l}1.32 \\
(0.95-1.83) \\
\end{array}$ & 23 & 189 & $\begin{array}{l}1.02 \\
(0.76-1.38) \\
\end{array}$ & 50 & 384 & $\begin{array}{l}1.14 \\
(0.92-1.40) \\
\end{array}$ & 73 \\
\hline Prostate & $(177)$ & 144 & $\begin{array}{l}0.85 \\
(0.60-1.21)\end{array}$ & 24 & 9489 & $\begin{array}{l}0.98 \\
(0.95-1.01)\end{array}$ & 3047 & 9633 & $\begin{array}{l}0.98 \\
(0.95-1.02)\end{array}$ & 3071 \\
\hline Testis & $(178)$ & 2857 & $\begin{array}{l}\mathbf{0 . 8 7} \\
(0.80-0.94)\end{array}$ & 393 & 139 & $\begin{array}{l}0.96 \\
(0.75-1.22)\end{array}$ & 38 & 2996 & $\begin{array}{l}\mathbf{0 . 8 7} \\
(0.81-0.94)\end{array}$ & 431 \\
\hline Other male genital & (179) & 114 & $\begin{array}{l}1.08 \\
(0.80-1.46)\end{array}$ & 17 & 145 & $\begin{array}{l}1.16 \\
(0.91-1.49)\end{array}$ & 34 & 259 & $\begin{array}{l}1.14 \\
(0.95-1.36)\end{array}$ & 51 \\
\hline Kidney & $(180)$ & 1112 & $\begin{array}{l}1.01 \\
(0.86-1.19)\end{array}$ & 159 & 1460 & $\begin{array}{l}0.99 \\
(0.88-1.11)\end{array}$ & 440 & 2572 & $\begin{array}{l}0.99 \\
(0.91-1.08)\end{array}$ & 599 \\
\hline Urinary bladder & $(181)$ & 834 & $\begin{array}{l}1.04 \\
(0.88-1.22)\end{array}$ & 141 & 2295 & $\begin{array}{l}0.94 \\
(0.87-1.02)\end{array}$ & 751 & 3129 & $\begin{array}{l}0.96 \\
(0.89-1.03)\end{array}$ & 892 \\
\hline Melanoma & $(190)$ & 4919 & $\begin{array}{l}0.85 \\
(0.78-0.93)\end{array}$ & 822 & 2740 & $\begin{array}{l}0.96 \\
(0.88-1.04)\end{array}$ & 802 & 7659 & $\begin{array}{l}\mathbf{0 . 9 0} \\
(0.84-0.96)\end{array}$ & 1624 \\
\hline Squamous cell skin & (191) & 708 & $\begin{array}{l}0.98 \\
(0.80-1.21)\end{array}$ & 109 & 1433 & $\begin{array}{l}\mathbf{0 . 8 9} \\
(0.81-0.97)\end{array}$ & 514 & 2141 & $\begin{array}{l}\mathbf{0 . 9 0} \\
(0.82-0.99)\end{array}$ & 623 \\
\hline Eye & (192) & 480 & $\begin{array}{l}0.97 \\
(0.75-1.25)\end{array}$ & 60 & 181 & $\begin{array}{l}0.96 \\
(0.72-1.28)\end{array}$ & 53 & 661 & $\begin{array}{l}0.97 \\
(0.80-1.18)\end{array}$ & 113 \\
\hline Nervous system & (193) & 6286 & $\begin{array}{l}1.16 \\
(1.05-1.29)\end{array}$ & 658 & 2192 & $\begin{array}{l}1.06 \\
(0.96-1.16)\end{array}$ & 564 & 8478 & $\begin{array}{l}\mathbf{1 . 1 3} \\
(1.05-1.21)\end{array}$ & 1222 \\
\hline Thyroid gland & (194) & 1496 & $\begin{array}{l}1.00 \\
(0.87-1.14)\end{array}$ & 219 & 356 & $\begin{array}{l}1.12 \\
(0.89-1.41) \\
\end{array}$ & 89 & 1852 & $\begin{array}{l}1.03 \\
(0.91-1.16) \\
\end{array}$ & 308 \\
\hline Endocrine glands & (195) & 1718 & $\begin{array}{l}1.00 \\
(0.88-1.14) \\
\end{array}$ & 256 & 1189 & $\begin{array}{l}1.00 \\
(0.88-1.13)\end{array}$ & 331 & 2907 & $\begin{array}{l}1.01 \\
(0.92-1.10) \\
\end{array}$ & 587 \\
\hline Bone & (196) & 821 & $\begin{array}{l}1.04 \\
(0.84-1.29)\end{array}$ & 91 & 69 & $\begin{array}{l}1.12 \\
(0.71-2.17)\end{array}$ & 15 & 890 & $\begin{array}{l}1.08 \\
(0.89-1.31)\end{array}$ & 106 \\
\hline Connective tissue & $(197)$ & 1013 & $\begin{array}{l}0.96 \\
(0.81-1.14)\end{array}$ & 135 & 318 & $\begin{array}{l}0.94 \\
(0.76-1.17)\end{array}$ & 97 & 1331 & $\begin{array}{l}0.95 \\
(0.83-1.09)\end{array}$ & 232 \\
\hline Non-Hodgkin lymphoma & $(200,202)$ & 2163 & $\begin{array}{l}1.00 \\
(0.85-1.18)\end{array}$ & 282 & 2038 & $\begin{array}{l}0.97 \\
(0.88-1.07)\end{array}$ & 602 & 4201 & $\begin{array}{l}0.98 \\
(0.90-1.07)\end{array}$ & 884 \\
\hline Hodgkin lymphoma & $(201)$ & 1736 & $\begin{array}{l}0.94 \\
(0.81-1.09)\end{array}$ & 212 & 141 & $\begin{array}{l}0.86 \\
(0.62-1.20)\end{array}$ & 44 & 1877 & $\begin{array}{l}0.93 \\
(0.81-1.07)\end{array}$ & 256 \\
\hline Myeloma & (203) & 211 & $\begin{array}{l}1.32 \\
(0.89-1.94) \\
\end{array}$ & 25 & 660 & $\begin{array}{l}0.96 \\
(0.82-1.11) \\
\end{array}$ & 207 & 871 & $\begin{array}{l}1.00 \\
(0.87-1.15)\end{array}$ & 232 \\
\hline Leukaemia & $(204-209)$ & 3775 & $\begin{array}{l}\mathbf{1 . 3 0} \\
(1.07-1.59)\end{array}$ & 343 & 1429 & $\begin{array}{l}1.06 \\
(0.95-1.18)\end{array}$ & 397 & 5204 & $\begin{array}{l}\mathbf{1 . 2 0} \\
(1.08-1.33)\end{array}$ & 740 \\
\hline Other and unspecified sites & (other) & 707 & $\begin{array}{l}1.00 \\
(0.81-1.24)\end{array}$ & 97 & 1766 & $\begin{array}{l}0.90 \\
(0.80-1.03)\end{array}$ & 577 & 2473 & $\begin{array}{l}0.93 \\
(0.84-1.02)\end{array}$ & 674 \\
\hline Any site & (any) & 50261 & $\begin{array}{l}0.97 \\
(0.94-1.00)\end{array}$ & 7208 & 59426 & $\begin{array}{l}0.99 \\
(0.94-1.04)\end{array}$ & 18001 & 109687 & $\begin{array}{l}0.99 \\
(0.96-1.02)\end{array}$ & 25209 \\
\hline
\end{tabular}

Reference group: one-child families. Bold type, $95 \% \mathrm{Cl}$ does not include 1.00. Poisson regression adjusted for age, sex, period, region, socioeconomic status. Ref: reference, $\mathrm{N}$ : number of cases 
Table 5 Relative risks for cancer of the thyroid gland

\begin{tabular}{|c|c|c|c|c|c|c|}
\hline \multirow[b]{2}{*}{ Cancer site } & \multicolumn{3}{|l|}{ All } & \multicolumn{3}{|l|}{ All } \\
\hline & $\mathrm{N}$ & $\begin{array}{l}\text { Birth order * } \\
\text { RR }(95 \% \mathrm{Cl})\end{array}$ & $\begin{array}{l}\text { Ref } \\
\mathrm{N}\end{array}$ & $\mathrm{N}$ & $\begin{array}{l}\text { Family size ** } \\
\text { RR }(95 \% \mathrm{Cl})\end{array}$ & $\begin{array}{l}\text { Ref } \\
N\end{array}$ \\
\hline \multicolumn{7}{|l|}{ Thyroid gland } \\
\hline papillary & 397 & $\mathbf{0 . 8 1}(0.72-0.92)$ & 442 & 736 & $0.93(0.77-1.12)$ & 103 \\
\hline follicular & 58 & $\mathbf{0 . 7 0}(0.52-0.94)$ & 82 & 116 & $0.76(0.50-1.17)$ & 24 \\
\hline medullary & 20 & $0.81(0.50-1.32)$ & 23 & 37 & $0.92(0.49-1.74)$ & 6 \\
\hline other & 31 & $0.85(0.59-1.24)$ & 45 & 64 & $1.17(0.69-1.98)$ & 12 \\
\hline
\end{tabular}

Reference group: ${ }^{*}=$ first born child, ${ }^{* *}=$ one-child families. Bold type, $95 \% \mathrm{Cl}$ does not include 1.00. Poisson regression adjusted for age, sex, period, region, socioeconomic status.

Ref: reference, N: number of cases

endometrial cancer for age at diagnosis below age 50 (one child families: $\mathrm{RR}=0.65$; two child families: $\mathrm{RR}=$ 0.64; three or four child families: 0.72). In contrast, the relative risk in the older age group was negatively associated with family size.

\section{Discussion}

Our results show that there is an effect of family size and birth order on different cancer sites. A significantly decreased risk for testicular cancer by increasing birth order is in line with previously published results $[5,6]$. Also family size was associated with testicular cancer which has been suggested to be the result of parental subfertility [28].

Family size and birth order showed protective effects for melanoma, in agreement with previous results on socioeconomic status [15,29-31]. We observed a similar association for squamous cell carcinoma, which might be related to the same factors.

Risk for cancer of the nervous system was positively associated with family size which is also supported by previous findings [32]. The number of siblings correlated with the risk for cancer suggesting an infectious etiology [33]. Some subtypes of leukemia as acute lymphoblastic leukemia have their origin in viral or bacterial

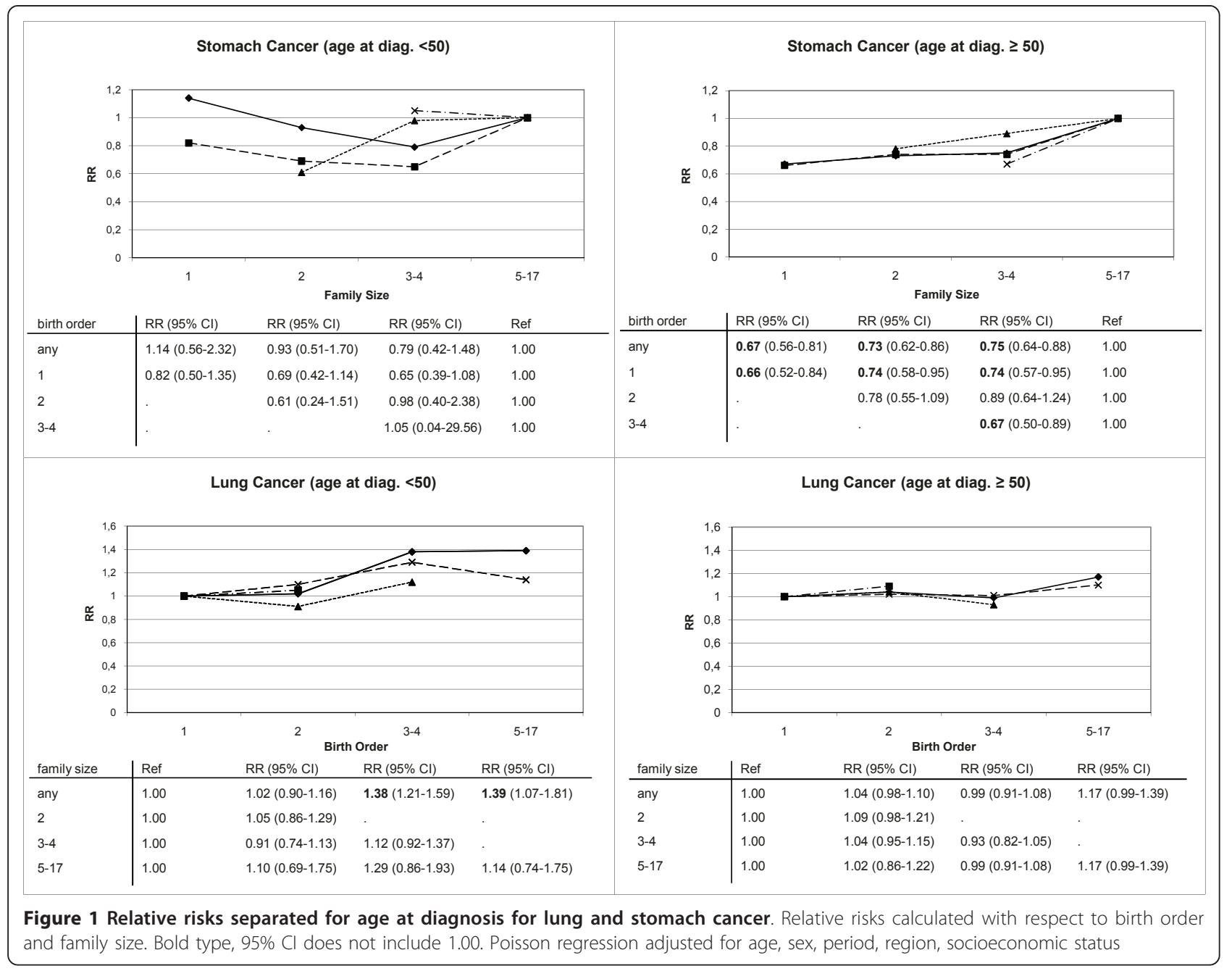




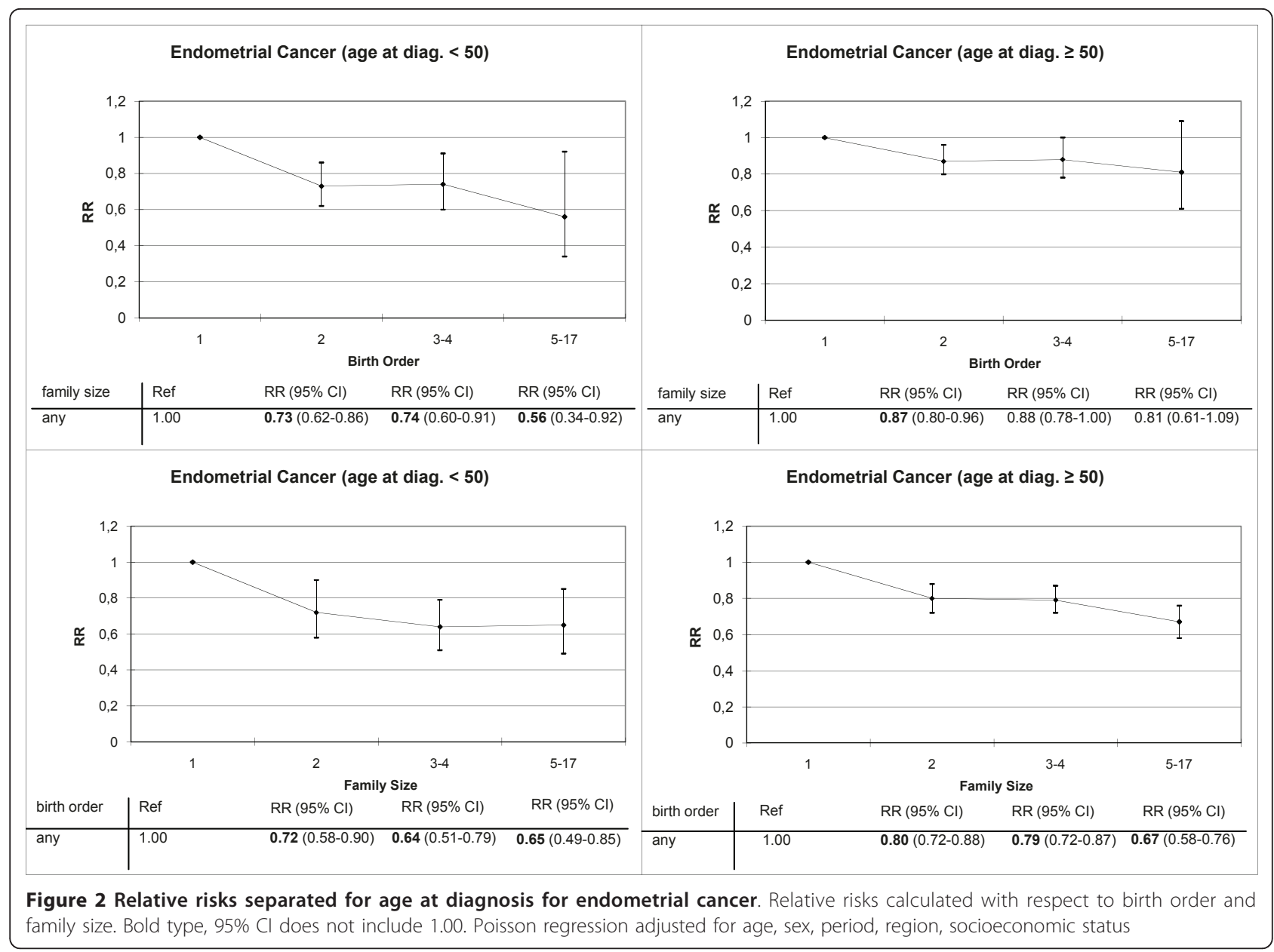

infection [34,35]. These can be shared easily in large families where all members have closer contact [36].

Family size influenced the risk of stomach cancer. Offspring in families with five or more children had an increased risk which has also been shown in previous studies [19,37]. Helicobacter pylori is an important cause of gastric cancer. The risk of infection is directly associated with sibship size [2,38-40]. As a consequence, members of larger families can easily share some infections.

Risk for lung cancer was increased in large families, most likely because of an association with low socioeconomic status [41,42]. An increasing risk with birth order and family size can clearly be seen for individuals diagnosed below age 50 . This might be due to the fact that the effect of birth order and family size is stronger in younger ages, where children still live with their parents and siblings. The effect of birth order and family size decreased from early to later adulthood.

Risk for endometrial cancer decreased with increasing birth order and family size. Family size showed a negative association for endometrial cancer in the group of people diagnosed before 50 years. This is in agreement with already published results reporting obesity as an important risk factor [21,43-45]. Obesity is associated with socioeconomic status [46,47], which may explain the decreased risk of large families with lower socioeconomic status [7]. Low birth order has been shown to be associated with obesity, especially in young women [48]. This might be explained by dietary habits depending on paternal resource.

Negative association between birth order and thyroid gland tumors is in line with already published results [14]. Nevertheless, more biological and epidemiological research is warranted to clarify the exact mechanisms through which higher birth order children have a decreased risk especially for subtypes as papillary and follicular thyroid gland tumors.

There are some limitations to our study. The information of smoking was not registered in our data. We were not able to include the smoking habits in our analysis. This information might have explained the association of lung cancer and birth order in a better way. As well as active, passive smoking can also have an effect 
on the risk of lung cancer. Lower birth order could lead to more passive smoking if older siblings smoke during the individual's childhood. Active smoking has an effect on the risk of cancer which cannot be taken into account in our analysis. Additionally, the information of obesity was not present in our data which could have helped us to explain effects on the risk of endometrial cancer.

\section{Conclusion}

Our results agree with already published findings on the influence of birth order and family size in melanoma. As these can be explained by the socioeconomic status this could also be an explanation for squamous cell carcinoma which shows an association of risk with birth order and family size. Our findings show that the risk of endometrial cancer is associated with birth order and family size. This can be explained by dietary habits that differ in families with varying number of children. Family size and birth order are associated with different cancer sites not only because of the effect of socioeconomic status. Taken together, our results suggest that the effect of birth order and family size decreases from early to later adulthood for some cancer sites as lung cancer and endometrial cancer.

\section{Abbreviations}

Cl: Confidence interval; RR: relative risk; ICD: International Classification of Disease.

\section{Acknowledgements}

Supported by Deutsche Krebshilfe, the Swedish Cancer Society, The Swedish Council for Working Life and Social Research, LSHC-CT-2004-503465. The Family-Cancer Database was created by linking registers maintained at Statistics Sweden and the Swedish Cancer Registry.

\section{Author details}

'Division of Molecular Genetic Epidemiology, German Cancer Research Center (DKFZ), Im Neuenheimer Feld 580, D-69120 Heidelberg, Germany. ${ }^{2}$ Center for Primary Health Care Research, Lund University, Malmö, Sweden. ${ }^{3}$ Stanford Prevention Research Center, Stanford University School of Medicine, California, USA.

\section{Authors' contributions}

MB carried out the statistical data analysis and wrote the manuscript. MW provided guidance and help in the preparation of the manuscript. HT commented on the manuscript. JS provided the data and commented on the manuscript. $\mathrm{KH}$ designed the study and commented on the analysis and the manuscript. All authors have accepted the final manuscript.

\section{Competing interests}

The authors declare that they have no competing interests.

Received: 15 March 2010 Accepted: 9 May 2011 Published: 9 May 2011

\section{References}

1. Michels KB, Trichopoulos D, Robins JM, Rosner BA, Manson JE, Hunter DJ, Colditz GA, Hankinson SE, Speizer FE, Willett WC: Birthweight as a risk factor for breast cancer. Lancet 1996, 348(9041):1542-1546.

2. Webb PM, Knight T, Greaves S, Wilson A, Newell DG, Elder J, Forman D: Relation between infection with Helicobacter pylori and living conditions in childhood: evidence for person to person transmission in early life. BMJ 1994, 308(6931):750-753.

3. Xu X, Dailey AB, Peoples-Sheps M, Talbott EO, Li N, Roth J: Birth weight as a risk factor for breast cancer: a meta-analysis of 18 epidemiological studies. J Womens Health (Larchmt) 2009, 18(8):1169-1178.

4. Westergaard T, Andersen PK, Pedersen JB, Frisch M, Olsen JH, Melbye M: Testicular cancer risk and maternal parity: a population-based cohort study. Br J Cancer 1998, 77(7):1180-1185.

5. Richiardi L, Akre O, Lambe M, Granath F, Montgomery SM, Ekbom A: Birth Order, Sibship Size, and Risk for Germ-Cell Testicular Cancer. Epidemiology 2004, 15:323-329.

6. Prener A, Hsieh CC, Engholm G, Trichopoulos D, Jensen OM: Birth order and risk of testicular cancer. Cancer Causes Control 1992, 3(3):265-272.

7. Hemminki K, Kyyrönen P: Parental age and risk of sporadic and familial cancer in offspring: implications for germ cell mutagenesis. Epidemiology 1999, 10:747-751.

8. Merrill RM, Fugal S, Novilla LB, Raphael MC: Cancer risk associated with early and late maternal age at first birth. Gynecol Oncol 2005, 96(3):583-593.

9. de Haan MC, Michels KB, Peeters PHM, van Noord PAH, Hennekam FAM, van der Schouw YT: Age of mother and grandmother in relation to a subject's breast cancer risk. Br J Cancer 2010, 102(9):1400-1404.

10. Zhang Y, Kreger BE, Dorgan JF, Cupples LA, Myers RH, Splansky GL, Schatzkin A, Ellison RC: Parental age at child's birth and son's risk of prostate cancer. The Framingham Study. Am J Epidemiol 1999, 150(11):1208-1212

11. Johnson K, Carozza SE, Chow EJ, Fox EE, Horel S, McLaughlin CC, Mueller BA, Puumala SE, Reynolds P, Behren JV, Spector LG: Parental age and risk of childhood cancer: a pooled analysis. Epidemiology 2009, 20(4):475-483

12. Sharpe CR, Franco EL, de Camargo B, Lopes LF, Barreto J, Johnsson R, Mauad M: The influence of parental age on the risk of Wilms' tumour. Paediatr Perinat Epidemiol 1999, 13(2):138-143.

13. Hemminki K, Kyyrönen $P$, Vaittinen P: Parental age as a risk factor of childhood leukemia and brain cancer in offspring. Epidemiology 1999, 10(3):271-275.

14. Hemminki K, Mutanen P: Birth order, family size, and the risk of cancer in young and middle-aged adults. Br J Cancer 2001, 84(11):1466-1471.

15. Hemminki $\mathrm{K}$, Zhang $\mathrm{H}$, Czene $\mathrm{K}$ : Socioeconomic factors in cancer in Sweden. Int J Cancer 2003, 105(5):692-700.

16. Weires M, Bermejo JL, Sundquist K, Sundquist J, Hemminki K: Socioeconomic status and overall and cause-specific mortality in Sweden. BMC Public Health 2008, 8:340.

17. Veierød MB, Adami HO, Lund E, Armstrong BK, Weiderpass E: Sun and solarium exposure and melanoma risk: effects of age, pigmentary characteristics, and nevi. Cancer Epidemiol Biomarkers Prev 2010, 19:111-120.

18. Nagore E, Hueso L, Botella-Estrada R, Alfaro-Rubio A, Serna I, Guallar J, González I, Ribes I, Guillen C: Smoking, sun exposure, number of nevi and previous neoplasias are risk factors for melanoma in older patients $(60$ years and over). J Eur Acad Dermatol Venereol 2010, 24:50-57.

19. Altieri A, Hemminki K: Number of siblings and the risk of solid tumours: a nation-wide study. Br J Cancer 2007, 96(11):1755-1759.

20. Ekberg-Aronsson M, Nilsson PM, Nilsson JA, Pehrsson K, Löfdahl CG: Socioeconomic status and lung cancer risk including histologic subtyping-a longitudinal study. Lung Cancer 2006, 51:21-29.

21. Shoff S, Newcomb P: Diabetes, body size and risk of endometrial cancer. Am J Epidemiol 1998, 148:234-40.

22. Larsson SC, Wolk A: Overweight, obesity and risk of liver cancer: a metaanalysis of cohort studies. Br J Cancer 2007, 97(7):1005-1008.

23. Larsson SC, Wolk A: Obesity and risk of non-Hodgkin's lymphoma: a meta-analysis. Int J Cancer 2007, 121(7):1564-1570.

24. Larsson SC, Wolk A: Body mass index and risk of multiple myeloma: a meta-analysis. Int J Cancer 2007, 121(11):2512-2516.

25. Hemminki K, Ji J, Brandt A, Mousavi SM, Sundquist J: The Swedish FamilyCancer Database 2009: prospects for histology-specific and immigrant studies. Int J Cancer 2010, 126(10):2259-2267.

26. Gao L, Weck MN, Raum E, Stegmaier C, Rothenbacher D, Brenner H: Sibship size, Helicobacter pylori infection and chronic atrophic gastritis: a population-based study among 9444 older adults from Germany. Int J Epidemiol 2010, 39:129-134 
27. WHO Organization WH: Manual of the international statistical classification of diseases, injuries and causes of death: seventh revision WHO. World Health Organization; 1957.

28. Aschim EL, Haugen TB, Tretli S, Grotmol T: Subfertility among parents of men diagnosed with testicular cancer. Int J Androl 2008, 31(6):588-594.

29. Pion IA, Rigel DS, Garfinkel L, Silverman MK, Kopf AW: Occupation and the risk of malignant melanoma. Cancer 1995, 75(2 Suppl):637-644.

30. Lee PY, Silverman MK, Rigel DS, Vossaert KA, Kopf AW, Bart RS, Garfinkel L, Levenstein MJ: Level of education and the risk of malignant melanoma. J Am Acad Dermatol 1992, 26:59-63.

31. Goodman KJ, Bible ML, London S, Mack TM: Proportional melanoma incidence and occupation among white males in Los Angeles County (California, United States). Cancer Causes Control 1995, 6(5):451-459.

32. Altieri A, Castro F, Bermejo JL, Hemminki K: Association between number of siblings and nervous system tumors suggests an infectious etiology. Neurology 2006, 67(11):1979-1983.

33. Shaw AK, Li P, Infante-Rivard C: Early infection and risk of childhood brain tumors (Canada). Cancer Causes Control 2006, 17(10):1267-1274.

34. zur Hausen $\mathrm{H}$ : Childhood leukemias and other hematopoietic malignancies: interdependence between an infectious event and chromosomal modifications. Int J Cancer 2009, 125(8):1764-1770.

35. Roman E, Simpson J, Ansell P, et al: Childhood acute lymphoblastic leukemia and infections in the first year of life: a report from the United Kingdom Childhood Cancer Study. Am J Epidemiol 2007, 165:496-504.

36. McNally RJQ, Eden TOB: An infectious aetiology for childhood acute leukaemia: a review of the evidence. Br J Haematol 2004, 127(3):243-263.

37. Vecchia CL, Ferraroni M, D'Avanzo B, Franceschi S, Decarli A, Baron JA: Number of siblings and subsequent gastric cancer risk. Eur J Cancer Prev 1995, 4:69-72.

38. Mendall MA, Goggin PM, Molineaux N, Levy J, Toosy T, Strachan D, Northfield TC: Childhood living conditions and Helicobacter pylori seropositivity in adult life. Lancet 1992, 339(8798):896-897.

39. Blaser MJ, Chyou PH, Nomura A: Age at establishment of Helicobacter pylori infection and gastric carcinoma, gastric ulcer, and duodenal ulcer risk. Cancer Res 1995, 55(3):562-565.

40. Koch A, Krause TG, Krogfelt K, Olsen OR, Fischer TK, Melbye M: Seroprevalence and risk factors for Helicobacter pylori infection in Greenlanders. Helicobacter 2005, 10(5):433-442.

41. Bernhardt EM: Fertility and Economic Status - Some Recent Findings on Differentials in Sweden. Population Studies 1972, 26(2):175-184.

42. Laaksonen M, Rahkonen O, Karvonen S, Lahelma E: Socioeconomic status and smoking: analysing inequalities with multiple indicators. Eur J Public Health 2005, 15(3):262-269.

43. Richardson LC, Thomas C, Bowman BA: Obesity and endometrial cancer: challenges for public health action. Womens Health (Lond Engl) 2009, 5(6):595-597.

44. Thomas CC, Wingo PA, Dolan MS, Lee NC, Richardson LC: Endometrial cancer risk among younger, overweight women. Obstet Gynecol 2009, 114:22-27.

45. Fader AN, Arriba LN, Frasure HE, von Gruenigen VE: Endometrial cancer and obesity: epidemiology, biomarkers, prevention and survivorship. Gynecol Oncol 2009, 114:121-127.

46. Rahkonen O, Lundberg O, Lahelma E, Huuhka M: Body mass and social class: a comparison of Finland and Sweden in the 1990s. J Public Health Policy 1998, 19:88-105.

47. Sundquist J, Johansson SE: The influence of socioeconomic status, ethnicity and lifestyle on body mass index in a longitudinal study. Int $J$ Epidemiol 1998, 27:57-63.

48. Koziel S, Kolodziej H: Birth order and BMI in teenage girls. Coll Antropol 2001, 25(2):555-560.

\section{Pre-publication history}

The pre-publication history for this paper can be accessed here: http://www.biomedcentral.com/1471-2407/11/163/prepub

\section{doi:10.1186/1471-2407-11-163}

Cite this article as: Bevier et al.: Influence of family size and birth order on risk of cancer: a population-based study. BMC Cancer 2011 11:163. 